\title{
INHERITANCE OF B-CHROMOSOMES IN PEARL MILLET
}

\author{
M. V. SUBBA RAO \\ Department of Botany, Andhra University, Waltair 530 003, India
}

Received 10.xii.79

\begin{abstract}
SUMMARY
The transmission pattern of three types of B-chromosome, Standard, Deficient and Iso, was studied through reciprocal crosses with non-B-carrying plants. The three types of B's were accumulated in the progeny when transmitted through the seed parent, probably as a result of the presence of directed segregation of the B's into the functional megaspore. Through the male side, Standard B's showed a slight gain whereas Deficient and Iso-B's showed reduced rates of transmission. It is suggested that a post-meiotic mechanism, viz., preferential fertilisation of eggs by male gametes having B's, might be responsible for the slight gain of Standard $B$ 's in the progeny when transmitted through the male side. It is suggested that the heterochromatic short arm is not responsible for the difference in the transmission frequencies of the three types of B's through the pollen parent and that the Deficient and Iso-B's might be subjected to gametic screening on the male side.
\end{abstract}

\section{INTRODUCTION}

SINCE the first report of the occurrence of B-chromosomes in pearl millet, Pennisetum americanum (L.) Leeke, $2 n=14$ (Pantulu, 1960), their structure and effects have been studied by Venkateswarlu and Pantulu (1970) and Pantulu and Manga (1975). Later Subba Rao and Pantulu (1978) obtained two types of B's (Deficient and Iso) from the original B-type (Standard) and studied their structure and effects on meiosis. The present study deals with the inheritance of these three types of B-chromosomes in pearl millet.

\section{MAterials AND MEthods}

Numerical variation was observed for all the three types of B's. For each plant one number of " $B$ " was found to be more frequent; the modal number was considered as the number of B's per plant. Plants having different modal numbers of B's of the three types were reciprocally crossed to plants without B's. For each B-class, the data have been obtained from a minimum of three reciprocal crosses and the pooled data are presented in the tables. The number of B-chromosomes was determined at pollen mother cell (PMC) meiosis from acetocarmine squash preparations. Similar studies were also made on plants having different combinations of Standard, Deficient and Iso-B's. The expected numbers of B-chromosomes in the progeny were calculated as if there were a regular $0-1,1-1,1-2$ and $2-2$ segregation of B's at anaphase $I$ in the parent plants with 1, 2, 3 and 4 B's respectively.

\section{Results}

The results on the transmission frequencies of the B's in the reciprocal crosses are presented in table 1. 


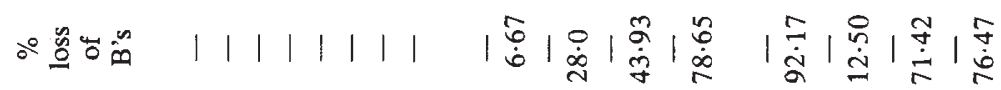

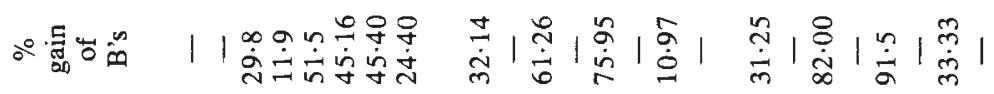
虫焉

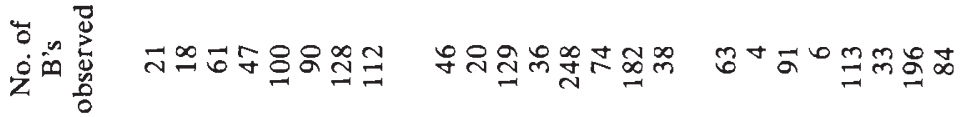

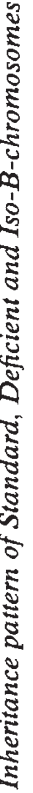

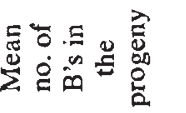
ถีทำกำสำ

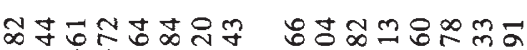

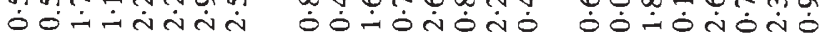

亗盇焉

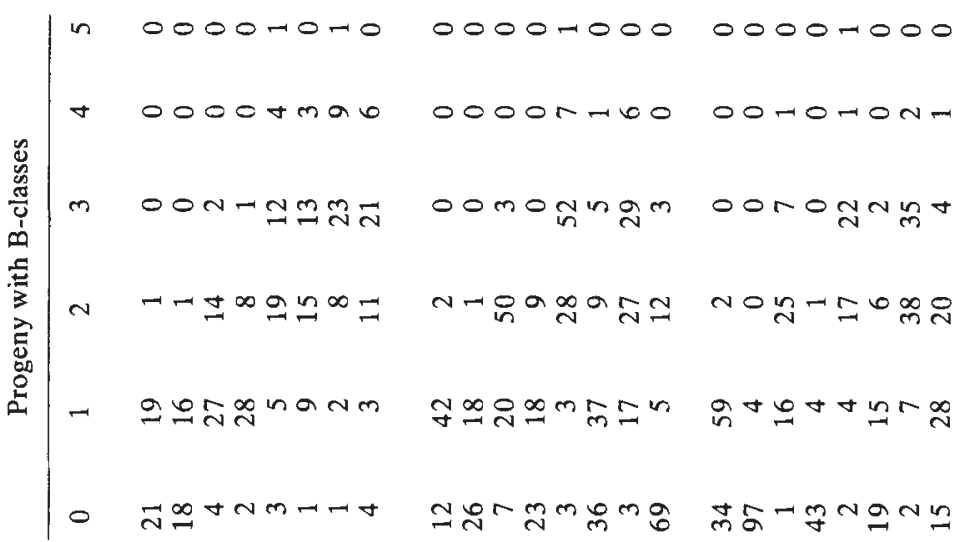

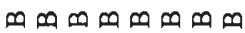
i⿱ ๓ாறாறாறாற

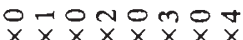
mm $m$

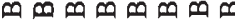
lonomogo honomoro honomoto

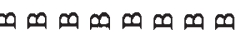

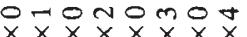
$\times \times \times \times \times \times \times$

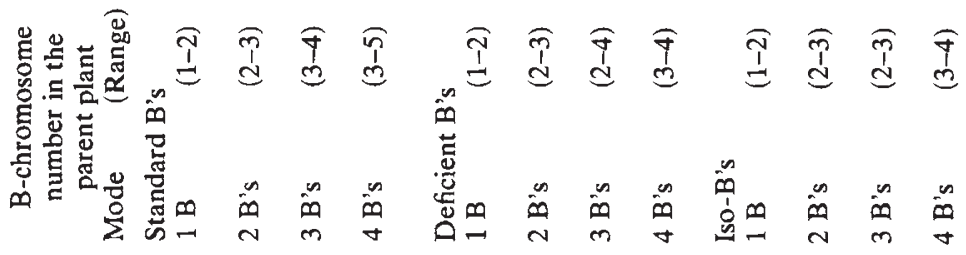




\section{(i) Inheritance of Standard B-chromosomes}

The reciprocal crosses involving 1 Standard $\mathrm{B}$ and $0 \mathrm{~B}$ did not differ with respect to the expected mean number of B's. In the progeny of both the crosses, an average of $0.51 \mathrm{~B}$-chromosomes per plant was observed. The result indicates that when there is one Standard B, it is transmitted with equal frequencies through males and females. Since the expected and observed numbers of B's in the progeny of both the reciprocal crosses were almost the same, it shows that there was neither loss nor gain of B's in the progeny. Two plants with 2 B's were observed in the progeny of both the reciprocal crosses. These might be the result of $\mathrm{B}$-chromosome nondisjunction at PMC meiosis. If this was the result of chromatid nondisjunction occurring during pollen grain mitoses and embryo mother cell mitoses, we might expect higher than the observed frequency of one in 40 and one in 35 plants respectively.

The inheritance of 2, 3 or 4 Standard B's followed a common pattern which was different from that observed for 1 Standard B. When 2, 3 or 4 Standard B's were used in the reciprocal crosses, gain of the B's was observed in the progeny. The reciprocal crosses differed only with respect to the percentage gain in all the three categories. In all the reciprocal crosses a higher percentage of gain was observed through the female than the male side.

The percentage gain of B's observed in the progeny in all the three categories can partly be accounted for by the anaphase I segregation in the parent plant. For example, when 2 Standard B's were transmitted through the female, there was a gain of 29.8 per cent of B's in the progeny. The data showed that there were fewer plants without B's than with 2 B's; hence, the overall increase in the number of B's may be due to a directed nondisjunction of B's into the functional megaspore. Similar situations have been observed for the transmission of 3 and 4 Standard B's through the female side.

When the plant with 2 Standard B's was used as the pollen parent, the total number of B's (47) in the progeny was slightly higher than the expected number (42), indicating a gain of 11.9 per cent. The overall increase in the number of B's in the progeny and the excess number of plants (19.0 per cent) with 2 B's over those without B's might be due to preferential fertilisation by the male gametes carrying $2 \mathrm{~B}$-chromosomes. A similar pattern has been observed for the transmission of 3 and 4 Standard B's through the male side.

\section{(ii) Inheritance of Deficient B's}

The Deficient B's showed differential transmission rates through the reciprocal crosses; there was accumulation of $\mathrm{B}$-chromosomes through the female side and loss through the male side; hence these differed from the Standard B's in their mode of transmission.

When the female carried 1 Deficient B-chromosome there was a gain of $32 \cdot 14$ per cent of B's in the progeny. The low frequency of plants with 0 B's in the progeny suggests that there might be preferential inclusion of B's in the functional megaspore. When the transmission was through the male side, there was a loss (6.67 per cent) of B's and plants without B's were more frequent than those with $1 \mathrm{~B}$. Loss of B's in the form of laggards either at 
telophase I or II or PMC meiosis was not observed in the parent plants. Hence, it is assumed that the pollen with B's may be at a disadvantage when compared with the pollen without B's and this might be responsible for the loss of B's in the progeny. The occurrence of progeny plants with 2 B's might be the result of numerical variation observed in the parent plant. A similar pattern of transmission was observed for 2, 3 and 4 Deficient B's in the reciprocal crosses.

\section{(iii) Inheritance of Iso-B's}

The mode of transmission of 1, 2, 3 and 4 Iso-B-chromosomes through both male and female parents followed, in general, a similar pattern to that observed for the Deficient B's. The only difference between these two types of B's was that the overall loss through the male side and gain through the female was comparatively more for the Iso-B's than for the Deficients.

In some of the reciprocal crosses involving Standard, Deficient or Iso-B's, progeny plants with numbers of B's higher than the modal number in the corresponding parents were observed. These plants might be the result of numerical variation and meiotic non-disjunction of B's in the parent plants.

\section{(iv) Inheritance of the B-chromosomes when present in different combinations}

To study the mutual effects of Standard, Deficient and Iso-B's on their transmission frequencies, reciprocal crosses were made between plants without B's and plants with different combinations of these three types of B's; the results are presented in table 2 . These results were compared with those observed when each of these three types of B's were present alone in corresponding numbers (table 1). From the tables it can be seen that irrespective of the B-combinations the three types of B's showed the same tendency of accumulation through the female side. On the male side, the Deficient and Iso-B's showed the same mode of transmission as was observed when they were present alone. Standard B's in combination with Iso-B's showed a decrease in their transmission frequency as revealed by the cross between $0 \mathrm{~B}$ plants (as female) and 1 Standard +1 Iso-B plants (as male).

\section{Discussion}

In the transmission frequency of the Standard B-chromosomes the reciprocal crosses differed with respect to the percentage gain of B's. Through the male side the Standard B's showed a slight gain. In this respect the present results are in agreement with those reported previously by Pantulu (1961). Further, he inferred the absence of any post-meiotic mechanism for the increase of B's on the male side and that the slight gain of B's in the progeny might be the result of non-disjunction during PMC meiosis. In the present study the occurrence of low frequencies of plants with 0 B's and of plants with B's in numbers equal to and higher than the parental number through the male side in the case of Standard B's, can partly be explained by the observed frequencies of different anaphase I distributions in the PMC's of the parent plant (Subba Rao, 1976); it also 
TABLE 2

Inheritance pattern of the Standard, Deficient and Iso-B-chromosomes when present in combinations

\begin{tabular}{|c|c|c|c|c|c|c|}
\hline & & & & & & \\
\hline & $\begin{array}{c}2 \mathrm{Sta} \\
2 \mathrm{Def} q\end{array}$ & $\underset{\times}{0 \mathrm{~B}} \stackrel{+}{ }$ & $\begin{array}{l}1 \mathrm{Sta} \\
1 \text { Iso } q\end{array}$ & $\underset{\times}{0 \mathrm{~B}} \stackrel{9}{ }$ & $\begin{array}{c}1 \text { Iso } \\
2 \text { Def } q\end{array}$ & $\underset{\times}{0 \mathrm{~B}} \stackrel{9}{\mathrm{P}}$ \\
\hline $\begin{array}{l}\text { Number of types of } \\
\text { B's in the progeny }\end{array}$ & $\stackrel{\times}{0} \mathrm{~B} \delta$ & $\begin{array}{l}2 \text { Sta } \\
2 \text { Def } \delta\end{array}$ & $0 \stackrel{\times}{\mathrm{B}}$ & $\begin{array}{l}1 \text { Sta } \\
1 \text { Iso o }\end{array}$ & $0 \mathrm{~B} \sigma$ & $\begin{array}{l}2 \text { Iso } \\
2 \text { Def } \delta\end{array}$ \\
\hline $0 \mathrm{~B}$ 's & 2 & 7 & 1 & 26 & 0 & 24 \\
\hline $1 \mathrm{Sta}$ & 3 & 3 & 4 & 1 & 0 & 0 \\
\hline $2 \mathrm{Sta}$ & 2 & 6 & 9 & 3 & 0 & 0 \\
\hline $3 \mathrm{Sta}$ & 5 & 1 & 0 & 0 & 0 & 0 \\
\hline 1 Def & 0 & 3 & 0 & 0 & 1 & 2 \\
\hline 2 Def & 7 & 1 & 0 & 0 & 7 & 0 \\
\hline 3 Def & 2 & 0 & 0 & 0 & 8 & 0 \\
\hline 4 Def & 0 & 0 & 0 & 0 & 1 & 0 \\
\hline 1 Iso & 0 & 1 & 7 & 0 & 6 & 2 \\
\hline 2 Iso & 0 & 0 & 2 & 0 & 4 & 0 \\
\hline $1 \mathrm{Sta}+1 \mathrm{Def}$ & 4 & 3 & 0 & 0 & 0 & 0 \\
\hline $1 \mathrm{Sta}+3 \mathrm{Def}$ & 1 & 0 & 0 & 0 & 0 & 0 \\
\hline $2 \mathrm{Sta}+1 \mathrm{Def}$ & 1 & 0 & 0 & 0 & 0 & 0 \\
\hline $2 \mathrm{Sta}+2 \mathrm{Def}$ & 3 & 2 & 0 & 0 & 0 & 0 \\
\hline 1 Iso + 1 Sta & 0 & 0 & 4 & 2 & 0 & 0 \\
\hline 2 Iso +1 Sta & 0 & 0 & 1 & 0 & 0 & 0 \\
\hline 1 Iso +2 Sta & 1 & 0 & 0 & 0 & 0 & 0 \\
\hline 1 Iso +1 Def & 0 & 0 & 0 & 0 & 1 & 1 \\
\hline Total no. of plants studied & 31 & 27 & 28 & 32 & 28 & 29 \\
\hline Mean no. of Standard B's & $1 \cdot 118$ & 0.9258 & 0.96 & $0 \cdot 2813$ & - & - \\
\hline Mean no. of Deficient B's & $1 \cdot 12$ & 0.444 & - & - & $1 \cdot 57$ & $0 \cdot 1034$ \\
\hline Mean no. of Iso-B's & - & - & $0 \cdot 61$ & 0.0625 & $0 \cdot 53$ & $0 \cdot 1034$ \\
\hline Gain of Standard B's & $19 \cdot 35 \%$ & - & $92.85 \%$ & - & - & - \\
\hline Gain of Deficient B's & $12.9 \%$ & - & - & - & $57 \cdot 1 \%$ & - \\
\hline Gain of Iso-B's & - & - & $21.42 \%$ & - & $7 \cdot 1 \%$ & - \\
\hline Loss of Standard B's & - & $7 \cdot 4 \%$ & - & $71 \cdot 85 \%$ & - & - \\
\hline Loss of Deficient B's & - & $51 \cdot 8 \%$ & - & - & - & $89 \cdot 6 \%$ \\
\hline Loss of Iso-B's & - & - & - & $87 \cdot 5 \%$ & - & $80 \cdot 0 \%$ \\
\hline
\end{tabular}

suggests the presence of a post-meiotic mechanism for the increase and accumulation of Standard B's in the progeny. This post-meiotic mechanism might be in the form of preferential fertilisation of the eggs by the B-carrying male gametes. This difference between the previous observations by Pantulu (1961) and the present study may be attributed to the genetic background in which the B's are present, i.e., the B's studied by Pantulu were present in the Sudanese stocks whereas those of the present study were transferred into a local inbred line (Subba Rao and Pantulu, 1978). The occurrence of preferential fertilisation was also inferred in maize by Blackwood (1956) and Catcheside (1956).

Differential transmission of B's in the reciprocal crosses and greater transmission frequency through the female side was reported in Lilium callosum (Kayano, 1956), Trillium grandiflorum (Rutishauser, 1956), Myrmeleotettix maculatus (Hewitt, 1973) and Melanoplus femur-rubrum (Leucov and Nur, 1973).

Deficient and Iso-B's differ in the presence and absence of the heterochromatic short arms respectively, but show similar transmission frequencies. This precludes any direct role for the short arm in the regulatory 
mechanisms concerned with transmission of B's. Further, with respect to the transmission frequencies on the male side, both Deficient and Iso-B's were different from the Standard B's. This difference is probably the result of the intact euchromatic long arms which in the Deficients and Iso's suffered a deficiency extending over two chromomeres.

Deficient and Iso-B's seem to be subjected to gametic screening on the male side, i.e., pollen with $0 \mathrm{~B}$ is more successful though occasionally pollen with low numbers of B's may function.

Acknowledgements.-The author is thankful to Dr J. V. Pantulu, Emeritus Professor, Department of Botany, Andhra University, Waltair (India), for his helpful discussions during the course of this work.

\section{REFERENCES}

BLACKWOOD. M. 1956. The inheritance of B-chromosomes in Zea mays. Heredity, 10, 353-366.

CATCHESIDE, D. G. 1956. The genetics of B-chromosomes in maize. Heredity, 10, 345-351. HEWITT, G. 1973. Variable transmission rates of B-chromosomes in Myrmeleotettix maculatus (Acrididae: Orthoptera). Chromosoma (Berl.), 40, 83-106.

KAYANO, H. 1956. Mem. Fac. Sci. Kyushu Univ., Ser. E2. 53. cited by Jones, R. N. 1975. B-chromosome systems in flowering plant and animal species. Int. Rev. Cytol., 40, 1-100.

LEUCOV, Z., AND NUR, U. 1973. Accumulation of B-chromosomes and preferential segregation in females of the grasshopper Melanoplus femur-rubrum. Chromosoma (Berl.), 42, 289-306.

Pantulu, J. V. 1960. Accessory chromosomes in Pennisetum typhoides. Curr. Sci., 29, 28-29.

PANTulu, J. V. 1961. Cytological studies in the genus Pennisetum with some cytological observations in the genus Cassia. Ph.D. thesis submitted to Andhra University, Waltair (India).

PANTULU, J. V., AND MANGA, V. 1975. Influence of B-chromosomes on meiosis in pearl millet. Genetica, 45, 237-251.

RUTISHAUSER, A. 1956. Genetics of fragment chromosomes in Trillium grandiflorum. Heredity, 10, 195-204.

SUBBA RAO, M. V. 1976. Cytogenetic studies of B-chromosomes and desynaptic mutants in pearl millet; Pennisetum typhoides S. \& H. Ph.D. thesis submitted to Andhra University, Waltair (India).

SUBBA RAO, M. V., AND PANTULU, J. V. 1978. The effects of derived B-chromosomes on meiosis in pearl millet, Pennisetum typhoides. Chromosoma (Berl.), 69, 121-130.

VENkATESwarlu, J., AND PANTUlu, J. V. 1970. The cytological behaviour of Bchromosomes in Pennisetum typhoides. Cytologia, 35, 444-448. 